\title{
Using Genetic Algorithm For Eye Location Detection
}

\author{
Ramadan M. Ramo \\ Department of Management Information Systems \\ College of Administration and Economics \\ University of Mosul
}

Received

29 / 09 / 2010
Accepted

14 / 04 / 2011

\begin{abstract}
الملخص

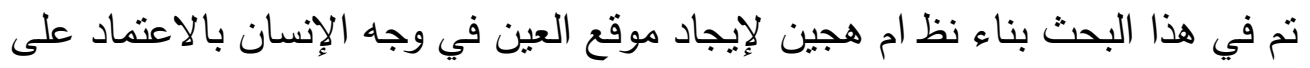

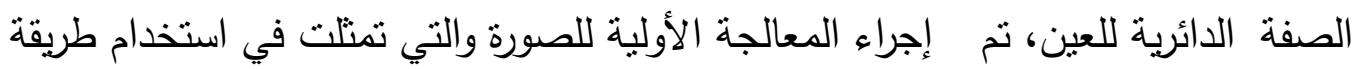

كاوسن Gaussian Smoothing Operator) لتنعيم الصورة وتقليل نسبة الضوضاء فيها، ومن ثم طريقة سوبل (Sobel Operator) لكثف الحواف، والطريقة المقترحة هي تهجين

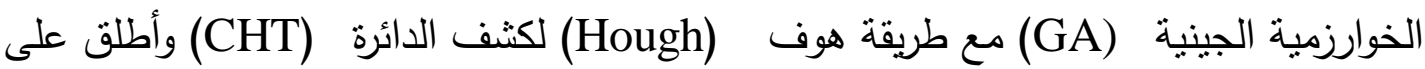
الطريقة الجديدة (GCHT)، وقد تم تطبيق الطريقة المقترحة على عدد من النماذج وظهر من

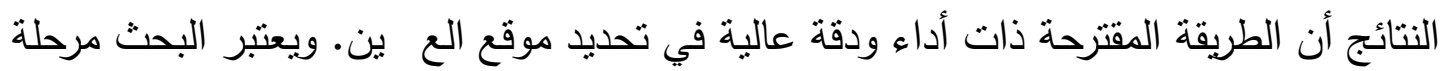

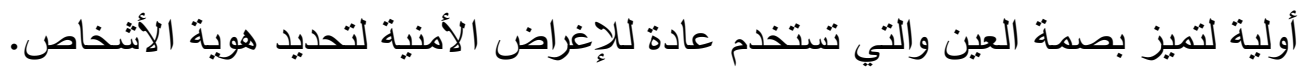

\section{Abstract}

In this research a hybrid system has been made to locate the position of eyes in the human face depending on the circular form of the eye. The primary process of image has been done using Gaussian Smoothing Operator to soften the image as well as to decrease the noise in it, then Sobel Operator has also been used to show the ridges. The suggested method is to hybridize the genetic algorithm (GA) together with Hough method to show the circle (CHT) which is recalled as (GCHT). This method has been applied on some models and the result shows that it has high accuracy and high performance in determining the eye position. The research is regarded as a primary level of recognition the for security purposes.

\section{Introduction}

There are Various techniques used for eye detection and have been proposed using texture, depth, shape and color information or 
combinations of those techniques. Focuses on several landmark points (eye corners, iris border points), [5] from which the approximate eyelid contours are estimated. The upper eyelid points are found using the observation that eye border pixels are significantly darker than surrounding skin and sclera. The detected eye boundary points are filtered to remove outliers and a polynomial curve which is fitted to the remaining boundary points. The lower lid is estimated from the known iris and eye. Two of the famous eye detection techniques are give below:

1. Projection function. [15]

2. Robust algorithm. [7]

\section{Edge Detection}

Edge detection is a fundamental tool used in most image processing applications to obtain information from the frames as a precursor step for feature extraction and object segmentation. This process detects the outlines of an object, boundaries between them and the background in the image. An edge-detection filter can also be used to improve the appearance of blurred or anti-aliased video streams. The basic edge-detection operator is a matrix-area gradient operator that determines the level of variance between different pixels. The edge detection operator is calculated by forming a matrix centered on a pixel chosen as the center of the matrix area. If the value of this matrix area is above a given threshold, then the middle pixel is classified as an edge. Examples of gradient-based edge detectors are Roberts, Prewitt, Canny and Sobel operators[16]. All the gradient-based algorithms have kernel operators that calculate the strength of the slope in directions which are orthogonal to each other, commonly vertical and horizontal. Later, the contributions of the different components of the slopes are combined to give the total value of the edge strength.[14]

\section{Image Smoothing}

Smoothing are used for blurring and for noise reduction. Blurring is used in preprocessing steps, such as removal of small details from an image prior to (large) object extraction, and bridging of small gaps in lines or curves. Noise reduction can be accomplished by blurring with a linear filter and also by nonlinear filtering [11]

\section{Circle Detection}

The HT can be extended by replacing the equation of the curve in the detection process. The equation of the curve can be given in an explicit or parametric form. In explicit form, the HT can be defined by considering the equation for a circle which is given by:

$\left(x-x_{0}\right)^{2}+\left(y-y_{0}\right)^{2}=r^{2}$ 
This equation defines a locus of points $(\mathrm{x}, \mathrm{y})$ centered on an origin $\left(\mathrm{x}_{0}, \mathrm{y}_{\mathrm{o}}\right)$ and with radius $\mathrm{r}$.This equation can again be visualized in two ways: as a locus of points $(\mathrm{x}, \mathrm{y})$ in an image, or as a locus of points $\left(\mathrm{x}_{0}, \mathrm{y}_{\mathrm{o}}\right)$ centered on $(\mathrm{x}, \mathrm{y})$ with radius $\mathrm{r}$. Figure 1 illustrates this dual definition. Each edge point in Figure 1 (a) defines a set of circles in the accumulator space. These circles are defined by all possible values of the radius and they are centered on the coordinates of the edge point. Figure 1 (b) shows three circles

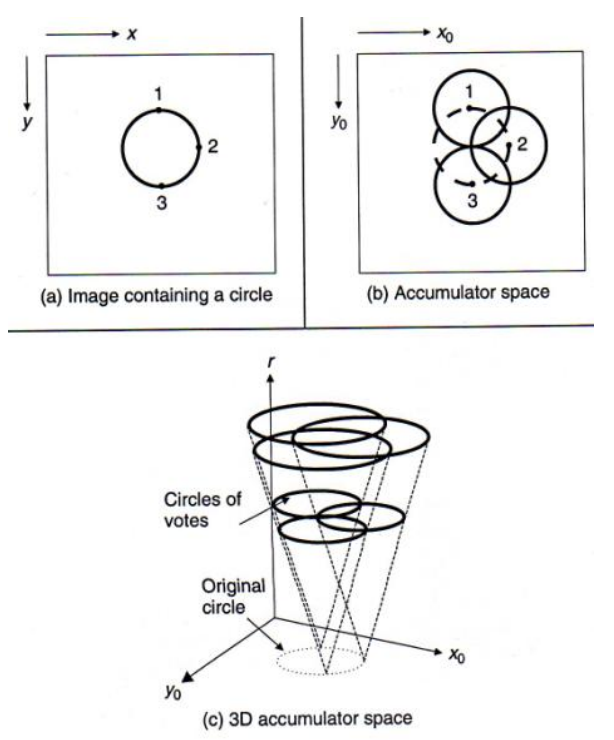

Figure 1 : Illustrating the Hough Transform for Circle

defined by three edge points. These circles are defined for a given radius value. Each edge point defines a circle for the other value of the radius. This implies that the accumulator space is three dimensional (for the three parameters of interest) and that edge points map to a cone of votes in the accumulator space. Figure 1 (c) illustrates this accumulator. After gathering evidence of all the edge points, the maximum in the accumulator space again corresponds to the parameters of the circle in the original image. The procedure of evidence gathering is the same as that for the HT for lines, but votes are generated in cones, according to Equation 1 can be defined in parametric form as:

$$
\begin{aligned}
& \mathrm{x}=\mathrm{x}_{0}+\mathrm{rcos}(\theta) \\
& \mathrm{y}=\mathrm{y}_{0}+\operatorname{rsin}(\theta)
\end{aligned}
$$

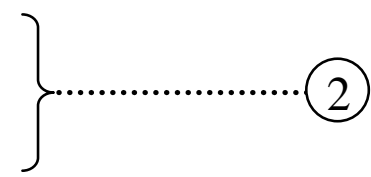

the advantage of this representation is that it allow us to solve for the parameters, thus, the HT mapping is defined by [8]:

$$
\begin{aligned}
& \mathrm{x}_{0}=\mathrm{x}-\mathrm{r} \cos (\theta) \\
& \mathrm{y}_{0}=\mathrm{y}-\mathrm{r} \sin (\theta)
\end{aligned}
$$

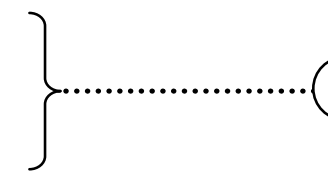




\section{Genetic Algorithms}

Genetic algorithms are general-purpose search algorithms based upon the principles of evolution observed in nature. Genetic algorithms combine selection, crossover, and mutation operators with the goal of finding the best solution to a problem. Genetic algorithms search for this optimal solution until a specified termination criterion is met.

The solution to a problem is called a chromosome. A chromosome is made up of a collection of genes which are simply the parameters to be optimized. A genetic algorithm creates an initial population (a collection of chromosomes), evaluates this population, then evolves the population through multiple generations in the search for a good solution for the problem at hand.[13] .

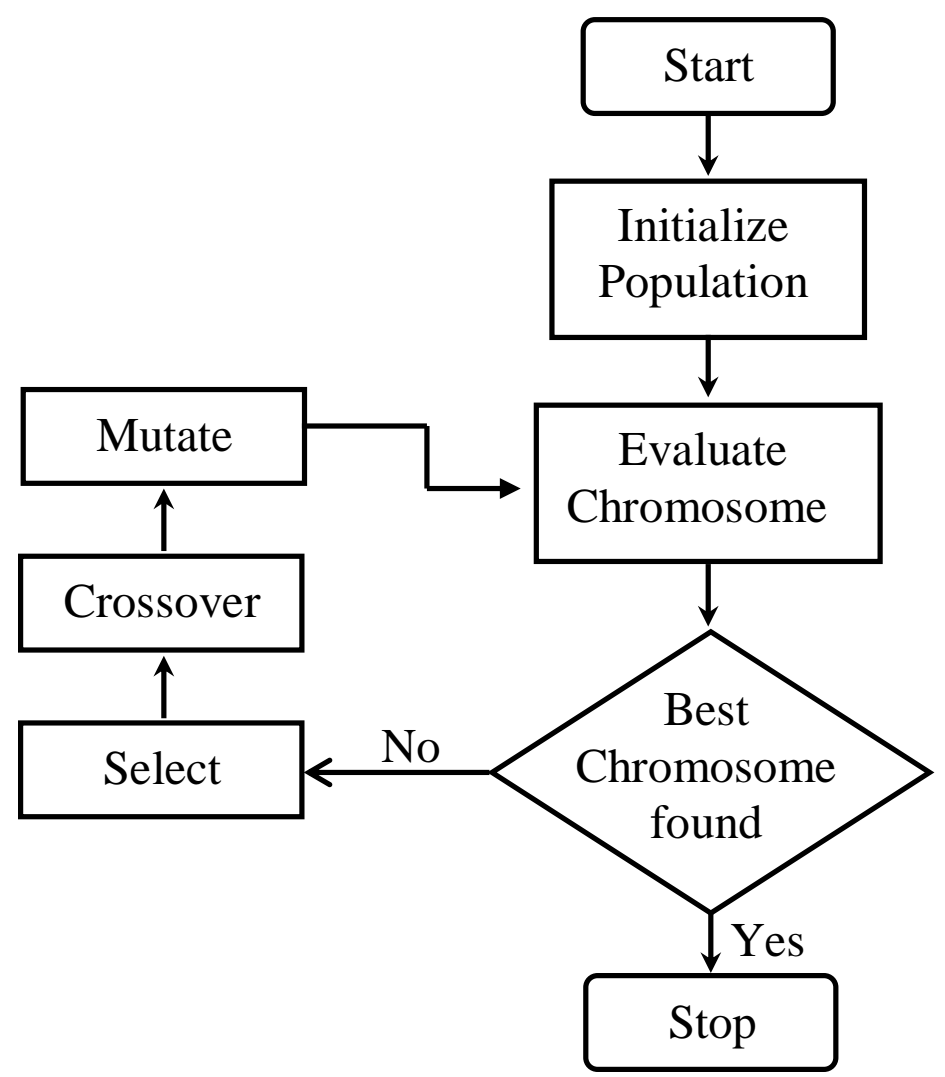

Figure 2: Flow Chart For Genetic Algorithm [1] [4]

\section{The Method Of Eye Location Detection}

The Following is the explanation of this method with its details.

\section{- Read The Required Information}

1. Read the image and put it in matrix (a).

2. Read the population size (popsize).

3. Read the maximum level for generations (genera). 


\section{- Convert The Image To Gray}

In this step, a formula will be performed to convert from RGB to gray color image.
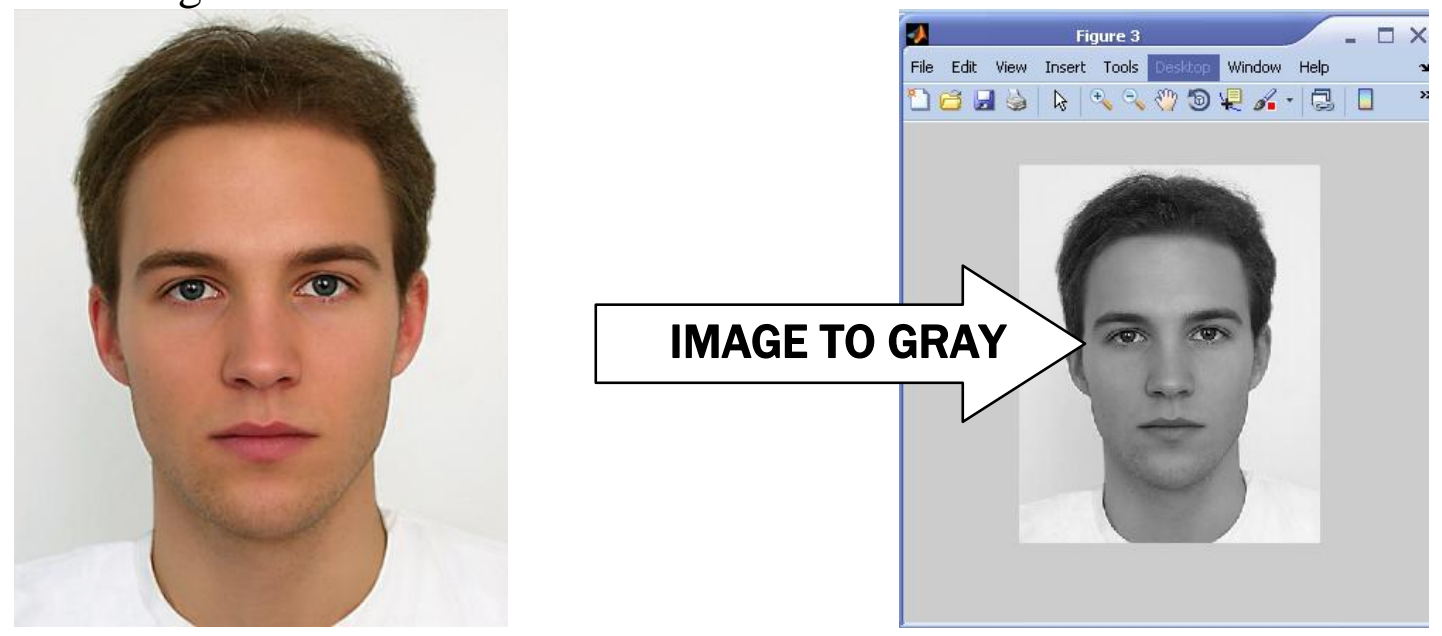

\section{- Image Smoothing}

The Gaussian smoothing operator is a 2-D convolution operator that is used to 'blur' images and remove detail and noise. In this sense it is similar to the mean filter, but it uses a different kernel that represents the shape of a Gaussian ('bell-shaped') hump. This kernel has some special properties which are detailed below. The Gaussian distribution in 1-D has the form

$$
G(x)=\frac{1}{\sqrt{2 \pi \sigma}} e^{-\frac{x^{2}}{2 \sigma^{2}}}
$$

The Gaussian distribution in 2-D has the form [12].

$$
G(x, y)=\frac{1}{\sqrt{2 \pi \sigma^{2}}} e^{-\frac{x^{2}+y^{2}}{2 \sigma^{2}}}
$$

Example of the Gaussian operator.
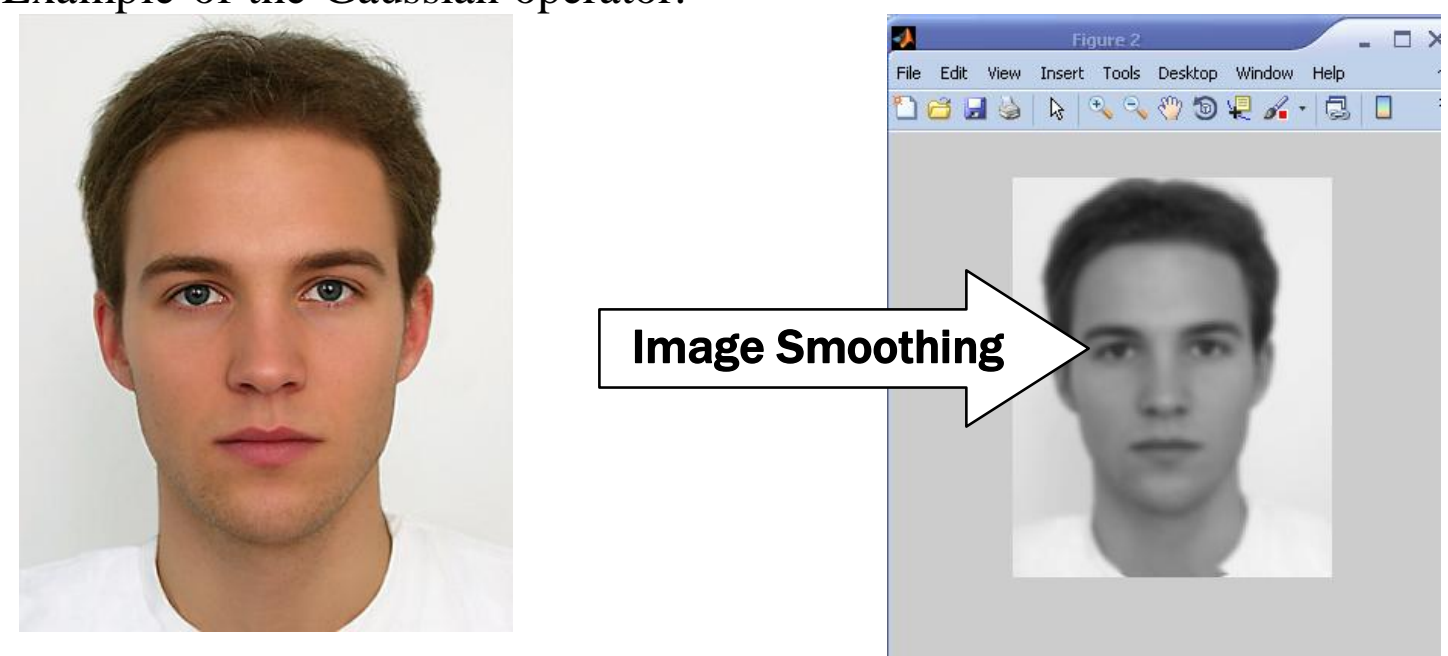


\section{- Edge Detection}

After smoothing the image and eliminating the noise, the next step is to find the edge strength by taking the gradient of the image. The Sobel operator performs a 2-D spatial gradient measurement on an image. The gradient magnitude is given by:

$$
|G|=\sqrt{G X^{2}+G Y^{2}}
$$

Then the approximate absolute gradient magnitude (edge strength) at each point can be found by the formula below which is simple to calculate compared to the above exact gradient magnitude. Approximate gradient magnitude given below:

$$
|G|=|G X|+|G Y|
$$

The Sobel operator uses a pair of $3 \times 3$ convolution masks, one estimating the gradient in the x-direction (Columns) and the other estimating the gradient in the y-direction (Row). Sobel Gx and Gy masks shown below each one estimate gradient $\mathrm{x}$ direction and $\mathrm{y}$ direction respectively. [10]

$$
\begin{gathered}
\mathrm{GX} \\
{\left[\begin{array}{lll}
-1 & 0 & +1 \\
-2 & 0 & +2 \\
-1 & 0 & +1
\end{array}\right]}
\end{gathered}
$$$$
\begin{array}{ccc}
\text { GY } & \\
{\left[\begin{array}{ccc}
-1 & -2 & -1 \\
0 & 0 & 0 \\
+1 & +2 & +1
\end{array}\right]}
\end{array}
$$

Example of the Sobel edge operator
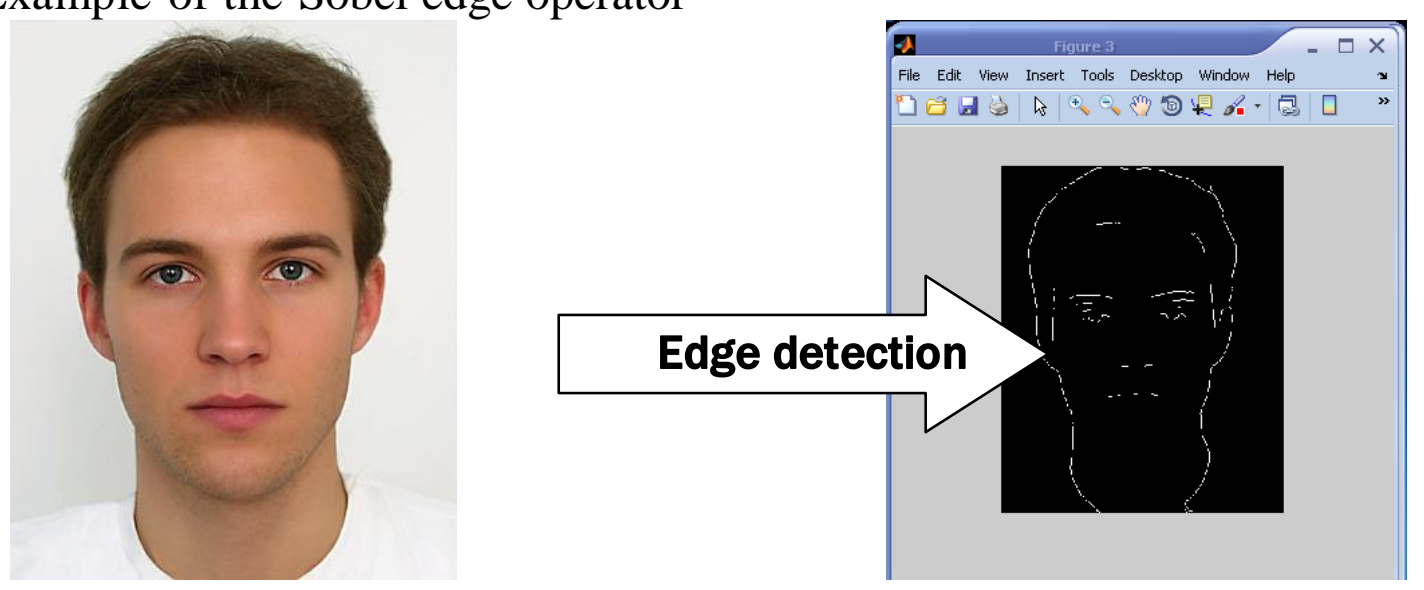

- The Application of Genetic Algorithms

. Population

The population consists of a number of individuals. The number of individuals is specified by the algorithm designer. If the size of the 
population is so large in some cases it could perform well for the Algorithm and it is the same case if the population is so small. Almost the best size between is 20-30 and sometimes between 50-100 to give best solution

Each individual (chromosome) consist of a number of values and their number is (specified) according to the case and the initial value is random within the limits of the case the intended case. Figure 3 shows the structure of the chromosome[2] [3].

In this method the chromosome contain three parts as in figure 4

\begin{tabular}{||l|l|l|l|l||}
\hline \hline Chromosome $=$ & Gene1 & gene2 & Gene3 & $\ldots . .$. \\
\hline \hline
\end{tabular}

Figure 3 : The structure of the Chromosome

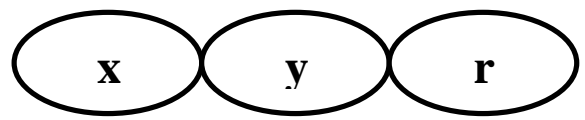

Figure 4 : The Chromosome in Method

\section{. Fitness Function}

In this stage, a combination between the equation of Hough circle with the genetic algorithm is specified for qualification, and the following equation represents the fitness function $\mathrm{F}(\mathrm{C})$, it gives maximal value when the point $\left(\mathrm{x}_{\mathrm{i}}, \mathrm{y}_{\mathrm{i}}\right)$ represent a shape in the image $\mathrm{E}$, and $\left(\mathrm{N}_{\mathrm{s}}\right)$ represent the population size.

$$
\mathrm{F}(\mathrm{C})=\frac{\sum_{i=0}^{N_{S}} E\left(x_{i}, y_{i}\right)}{N_{s}}
$$

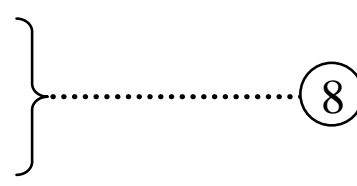

\section{. Evaluating Fitness Of Chromosomes}

Each chromosome is evaluated according to its fitness. Fitness means the performance of the chromosome to be a good solution. Fitness of the chromosome is defined by a fitness function.

\section{- Selection}

After evaluating each chromosome, selection takes place. According to Darwinian theory, the chromosome with higher fitness will survive. There are various methods for selection. We have used the most common Roulette-wheel Selection.

\section{- Crossover}

This operator which produces new chromosome by taking some parts of the old chromosome to get new chromosome called "child". There are several types of crossover such as: (one-point crossover, twopoint crossover, uniform crossover, arithmetic crossover, heuristic crossover[9]. In this research we used the one point crossover. The following figure explain that 


\begin{tabular}{|l|l|l|l|l|l|l|l|}
\hline Parent 1: & 1 & 0 & 0 & 1 & $:$ & 0 & 1 \\
\hline Parent 2: & 0 & 1 & 0 & 0 & $:$ & 1 & 1 \\
\hline
\end{tabular}

\begin{tabular}{|l|l|l|l|l|l|l|l|}
\hline Child 1 : & 1 & 0 & 0 & 1 & $:$ & 1 & 1 \\
\hline Child 2 : & 0 & 1 & 0 & 0 & $:$ & 0 & 1 \\
\hline
\end{tabular}

Figure 5 : One Point Crossover

\section{. Mutation}

Mutation means changing one or more genes in a chromosome, with these new gene values, the genetic algorithm may be able to arrive to at better solution than was previously possible, there are several types of mutation such as (flip bit, boundary, Gaussian). In this research we used the flip bit[6]. The follow figure represent new mutation.

Mutation
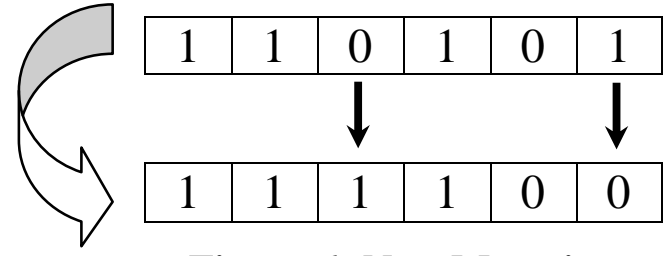

Figure 6: New Mutation

\section{Flow Chart Of The Method}

The following diagram (7) shows the steps.<smiles>C1CCCCCC1</smiles>

Enter image

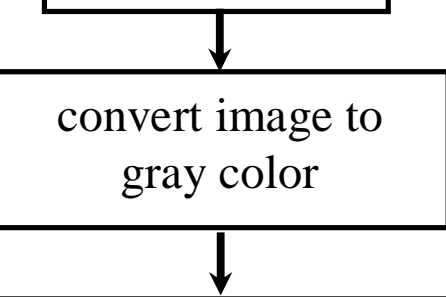

perform smoothing operator

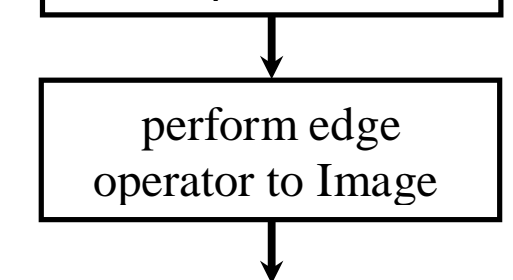

The execution of the hybrid suggested method 


\section{Experimental Results :}

The new algorithm has been applied on a set of images. This algorithm is an accurate way to locate the eye and it give good result to determine the eye position, such a result has been obtained after many level the image of $320 * 400$ has faced (changing image color into grey, Edge Detection, Smoothing, and applying the method).

\section{Image (1)}

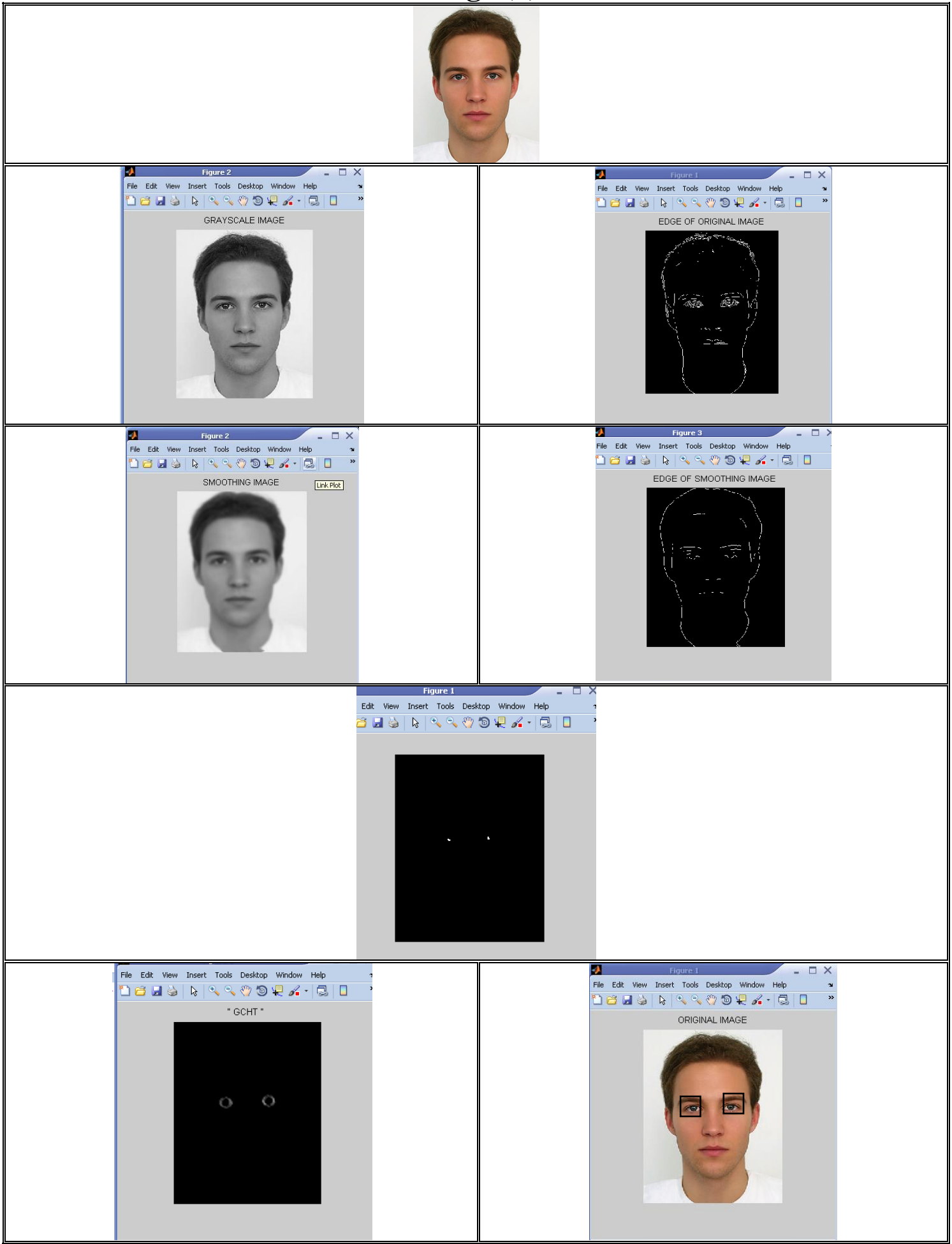


Using Genetic Algorithm For Eye Location Detection.

\section{Image (2)}

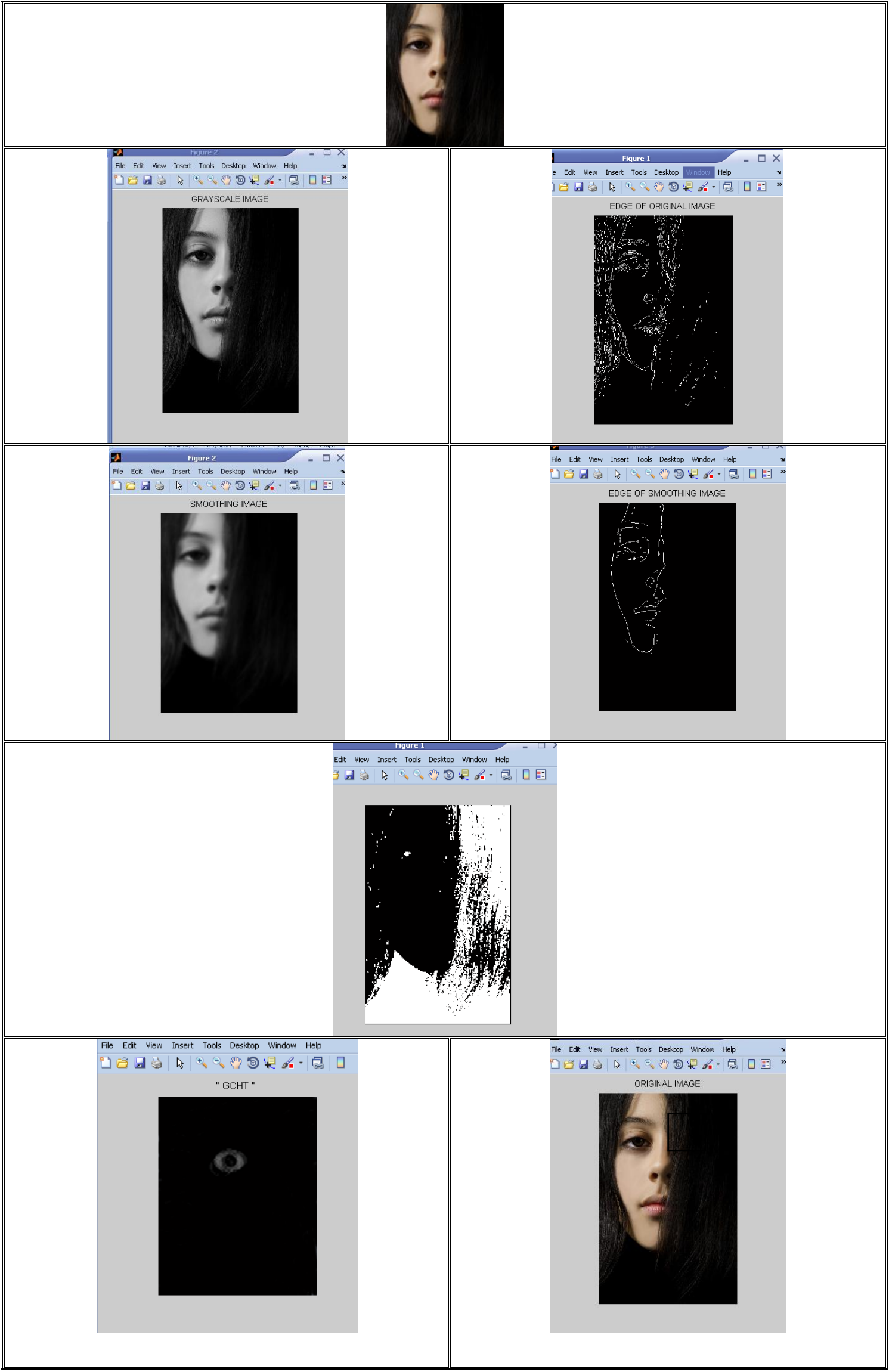




\section{Ramadan M. Ramo}

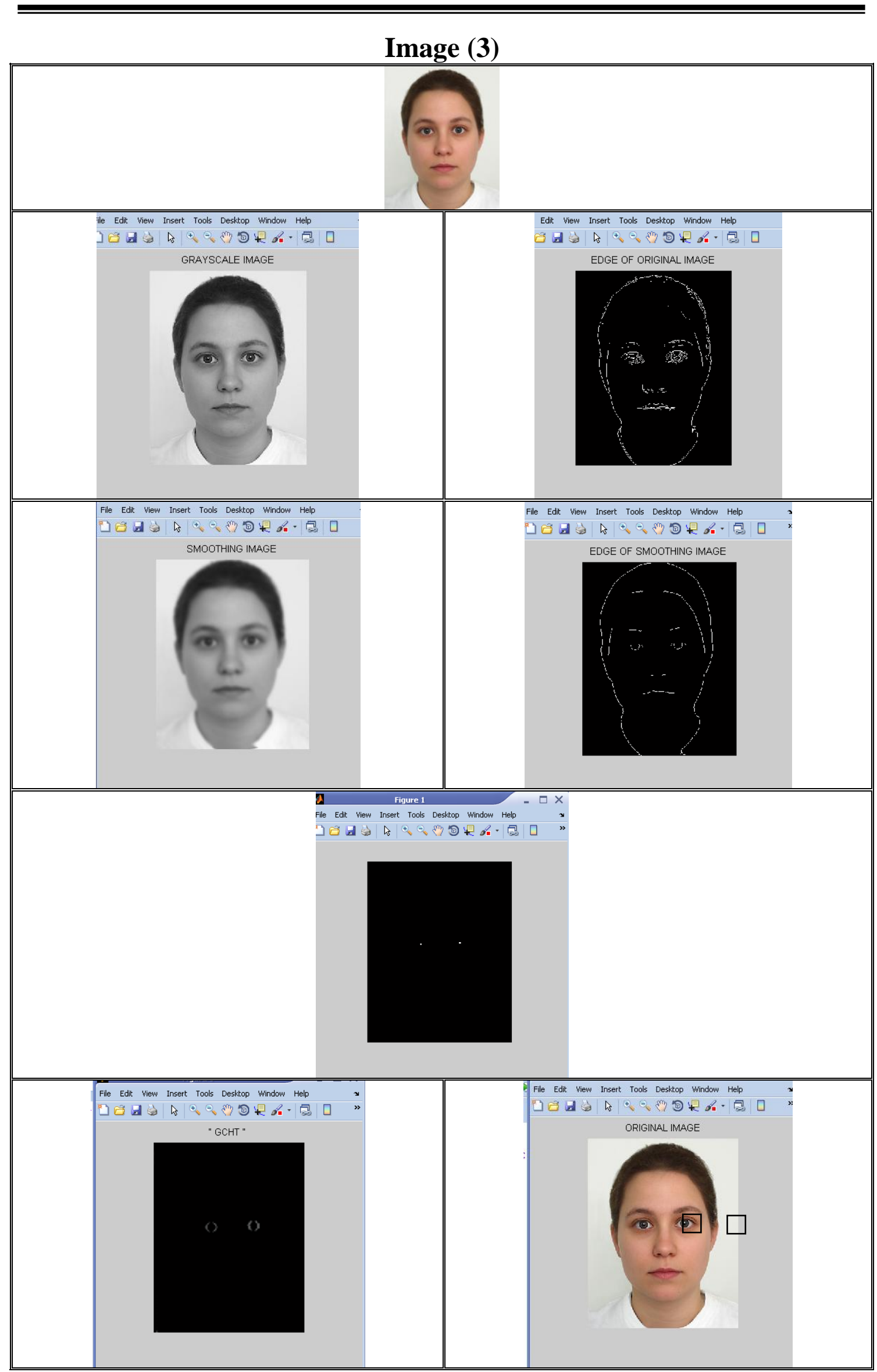




\section{Table For Eye Location}

The result in table (1) show the eye position of the image (both right and left side of the face), and also to determine those position depending on abscissas $(\mathrm{x}, \mathrm{y})$. The second example shows that the image doesn't have left eye, Thus, the result have no such abscissas.

Table (1)

\begin{tabular}{|c|c|c|c|c|c|}
\hline \multirow{2}{*}{ No. } & \multirow{2}{*}{ Image } & \multicolumn{2}{c|}{ Right eye } & \multicolumn{2}{c|}{ Left eye } \\
\cline { 3 - 6 } & & $\mathbf{X}$ & $\mathbf{Y}$ & $\mathbf{X}$ & $\mathbf{Y}$ \\
\hline Image (1) & 20 & 39 & 61 & 71 & 60 \\
\hline Image (2) & 20 & 97 & 112 & $\ldots \ldots \ldots .$. & $\ldots \ldots \ldots .$. \\
\hline Image (3) & 20 & 120 & 181 & 204 & 179 \\
\hline
\end{tabular}

\section{Conclusion and Future Works}

\section{Conclusion}

1. The proposed method in the search gave good results and acceptable, and has the speed to obtain the results.

2. The hybridization of genetic algorithm with the Hough Method represents a new tack in the direction of hybridization with gene algorithm methods to extracting characteristics.

\section{Future Works}

1. The application of the proposed algorithm with the addition of Fuzzy logic to increase the accuracy of the results.

2. The use of neural networks to discover the location of the eye. 3. The application of the proposed algorithm to detect the presence of a circular shape in the picture.

\section{References}

1) Ashish Narain \& Amit Srivastava ."Artificial Intelligence in Engineering", 2003.

2) Frontline Systems and Speadsheet Optimization, "What is a Genetic Evolutionary Algorithm", http://www.solver.com/gabasic.html.

3) Huda A. Al-Omari, "A Genetic Chromosomes for Image Segmentation", M.Sc Thesis, University of Mosul, 2004. 
4) G. S. Basheer, "using Genetic Algorithm for Image Maching", M.Sc. Thesis, University of Mosul, 2003.

5) Kaushal Kumar Dhruw, Aswin Kumar Tigga," Eye Detection Using Variants Of Hough Transform", a thesis submitted in partial fulfillment of the requirements for the degree of bachelor of technology in electronics \& Instrumentation Engineering, 2009.

6) Kiat C. chen \& Ian H. \& Cao A., " A Genetic Algorithm for Minimum Tetrahedralization of a Convex Polyhedron", Department of Computer Science, Memorial University of Newfoundland, St. John's, Newfoundland, Canada, kiat@cs.mun.ca, 2003.

7) Kun Peng, Liming Chen, Su Ruan, and Georgy Kukharev, "A Robust Algorithm for Eye Detection on Gray Intensity Face without Spectacles", Faculty of Computer Science and Information Technology, Technical University of Szczecin Zolnierska 49, 71210 Szczecin, Poland, 2005.

8) Mark S. Nixon \& Alberto S. Aguado "Feature Extraction and Image Processing second edition", Academic Press is an imprint of Elsevier Linacre House, Jordan Hill, Oxford OX2 8DP, UK 84 Theobald's Road, London WC1X 8RR, UK,2008.

9) Philipp Kohn, "Combining Genetic Algorithm and Neural Networks", M.Sc. Thesis, University of Tennessee, 1994

10) Rafael C. Gonzalez, Richard E. Woods, Steven L. Eddins, "Digital image processing using matlab", Prentice-Hall, Inc. Upper Saddle River, 2007.

11) Rafael C. Gonzalez, Richard E. Woods, "Digital Image Processing Second Edition", Prentice-Hall, Inc. Upper Saddle River, New Jersey 07458, 2002.

12) R. Fisher, S. Perkins, A. Walker and E. Wolfart, "image processing Learning resources",2003. http:// homepages.inf.ed.ac.uk/ rbf / HIPR2/ gsmooth.htm.

13) Roger L. Wainwright, "Introduction to Genetic Algorithms theory and application", Dept. of Mathematical and Computer Sciences, University of Tulsa, Copyright RLW, rogerw@penguin.mcs.utulsa.edu, 1993.

14) Scott E.Umbaugh, Ph.D., "computer vision and image processing", Prentice-Hall, Inc. A Simon \& Schuster Company, Upper Saddle River, 1998.

15) Zhi-Hua Zhou, Xin Geng, "Projection functions for eye detection", State Key Laboratory for Novel Software Technology, Nanjing University,2003.

16) Tinku Acharya \& Ajoy K. Ray, "Image Processing Principles and Applications", Electronics and Electrical Communication Engineering Department Indian Institute of Technology Kharagpur, India, 2005. 